\title{
Modelling and Optimization of Corrosion Penetration Rate (CPR) for Crude Oil Transportation Processes by Pipeline
}

\author{
Rania Ahmad Elrifai \\ Benghazi University, Industrial and Manufacturing \\ System Engineering \\ Benghazi, Libya
}

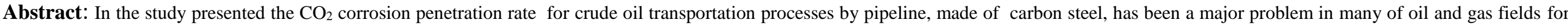

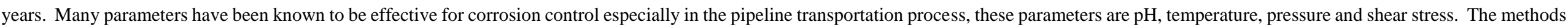

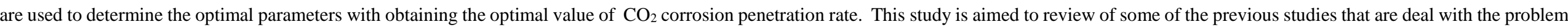

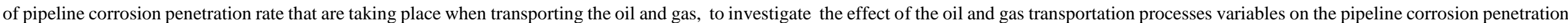

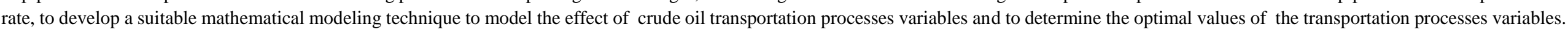

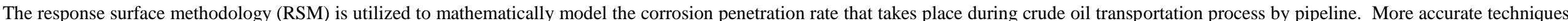

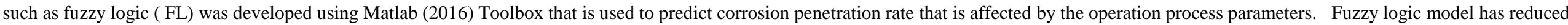

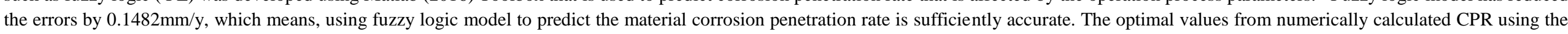
fuzzy logic model with the formula by using Root- Sum Square (RSS), were found that CPR value is $2.16 \mathrm{~mm} / \mathrm{y}$, the temperature is $44.4{ }^{\circ} \mathrm{C}$, pressure is $34.28 \mathrm{~Pa}$, $\mathrm{pH}$ is 5.51 and shear stress is 1 bar.
\end{abstract}

Keywords: $\mathrm{CO}_{2}$ corrosion penetration rate (CPR), Norsok -506 software, Response Surface Methodology, ANOVA, Fuzzy logic model.

\section{INTRODUCTION}

Corrosion of carbon steel is a significant problem in the oil and gas production and also their transportation process, which causes significant economic loss [1]. As a result of corrosion, rupture of the pipe wall frequently causes failure of petroleum and gas pipelines. The breakdowns are followed by large losses of the products, environmental pollution and ecological disasters [2]. The majority of oil and gas pipelines failures result from $\mathrm{CO}_{2}$ corrosion of carbon and low alloy steels [3]. It occurs at all stages of production from downhole to surface equipment and processing facilities [4]. The mechanism of carbon dioxide corrosion is a complicated process that is influenced by many factors and conditions temperature, $\mathrm{pH}$, a partial pressure of $\mathrm{CO}_{2}$, etc.)[5].
Oilfield corrosion manifests itself in several forms, among which $\mathrm{CO}_{2}$ corrosion (sweet corrosion) and hydrogen sulfide $\left(\mathrm{H}_{2} \mathrm{~S}\right)$ corrosion (sour corrosion) in the produced fluids and corrosion by dissolved carbon dioxide in water injection are the most prevalent forms of attack found in oil and gas production [6]. Filed data of main line sarir is selected as case study as to predict and reduce corrosion penetration rate. This study will be review several steps for obtaining the optimal solution which minimizes corrosion penetration rate $(\mathrm{mm} / \mathrm{y})$, through selection the optimum input parameters are temperature $\left({ }^{\circ} \mathrm{C}\right), \mathrm{CO}_{2}$ total pressure (bar), $\mathrm{pH}$ and shear stress $(\mathrm{Pa})$ with Norsok software is implemented to relate the corrosion penetration rate with the working conditions of transportation processes of the main line sarir. The Norsok -506 software for corrosion measurements were used as true values to introduce the capabilities of the predicted novel analysis methods. The prediction of CPR was carried out with two methods, 
Response Surface Method (RSM) and fuzzy Logic(FL) method, for the prediction and analysis of the effect of some selected parameters, namely, temperature, $\mathrm{pH}$, pressure, shear stress by the ANOVA. The defuzzification process in FL will select with two methods, the centroid and Root- Sum Square method. Root- Sum Square method will result in the corrosion penetration rate with explain the reason of selection this method. The developed fuzzy logic model will also test by comparing the results with the result using RSM technique. The comparison was carried out based on the mean absolute error between the predicted values and the actual values, Based on comparison between models that were developed by using fuzzy logic and RSM technique, it was concluded that, models based on fuzzy logic were better Mean Absolute Error (MAE) of 0.0412 with in comparison 0.168 for RSM.

\section{MATERIALS AND METHODS}

\subsection{Material}

The Work Material of Pipeline used in the present study is Grade API X52 refer as the following, API refers to American Petroleum Institute, the X symbol followed by a two or three digit number equal to the specified minimum yield strength in 1000 psi rounded down to the nearest integer, 52 refers to Minimum Yield at pipe body $360 \mathrm{MPa}(52,200$ psi). Basic pipeline data represent the following are pipeline length is $513 \mathrm{~km}$, pipe diameter is(outside diameter) 34in $(864 \mathrm{~mm}$ ) and wall thickness is $9.52 \mathrm{~mm}$, pipe type is seam welded/ spiral welded. The mechanical properties specified minimum yield strength is $358 \mathrm{MPa}$, specified ultimate tensile strength is $455 \mathrm{MPa}$ and coating by field applied tape wrap. Chemical properties of X52 pipeline with a composition of $0.16 \% \mathrm{C}$, $0.20 \% \mathrm{Cu}$ and $0.45 \% \mathrm{Si}, 1.10-1.60 \% \mathrm{Mn}$ as shown in Table 1 .

Table1:Chemical composition of X52 with thickness $(t) \leq 25$ mm

\begin{tabular}{|c|c|c|c|c|c|}
\hline $\mathbf{C}$ & 0.16 & $\mathbf{C u}$ & 0.20 & $\begin{array}{c}\mathbf{C r}+\mathbf{M o +} \mathbf{~ N i}+ \\
\mathbf{C u}\end{array}$ & 0.5 \\
\hline $\mathbf{S i}$ & 0.45 & $\mathbf{N}$ & 0.014 & $\mathbf{N b + V}$ & 0.1 \\
\hline $\mathbf{M n}$ & $1.10-1.60$ & $\mathbf{N b}$ & 0.04 & $\mathbf{N b +} \mathbf{V}+\mathbf{T i}$ & 0.12 \\
\hline $\mathbf{P}$ & 0.025 & $\mathbf{T i}$ & 0.02 & $\mathbf{C E V}(\mathbf{I I W})$ & $0.34^{2}$ \\
\hline $\mathbf{S}$ & 0.005 & $\mathbf{V}$ & 0.05 & $\mathbf{P C M}$ & $0.23^{2}$ \\
\hline
\end{tabular}

\begin{tabular}{|c|c|c|c|c|c|}
\hline $\mathbf{C r}$ & 0.20 & $\mathbf{A s}$ & 0.02 & $\mathbf{B i}$ & 0.010 \\
\hline $\mathrm{Mo}$ & 0.08 & $\mathrm{Sb}$ & 0.1 & $\mathrm{Ca}$ & 0.005 \\
\hline $\mathrm{Ni}$ & 0.20 & $\mathrm{Sn}$ & 0.015 & $\mathrm{~B}$ & 0.0005 \\
\hline $\mathrm{Al}$ & $0.020-0.060$ & $\mathrm{~Pb}$ & - & $\mathrm{Bi}$ & 0.010 \\
\hline
\end{tabular}

$\mathrm{CEV}=\mathrm{C}+\mathrm{Mn} / 6+(\mathrm{Cr}+\mathrm{Mo}+\mathrm{Cu}) / 15$ (1)

PCM=C+Si/30+(Mn+Cu+Cr)/20+Ni/60+ Mo/15+V/10+5B

In this study, an attempt is made to establish the input-output relationship of $\mathrm{CO}_{2}$ corrosion penetration rate (CPR) of the transportation processes parameters by pipeline. It is important to note that selection of the range of the operating parameters is an important consideration.

Table 2: Factors and selected for the experiments

\begin{tabular}{|c|c|c|c|c|c|}
\hline \multirow{2}{*}{ No } & \multirow{2}{*}{ Factors } & \multirow{2}{*}{ Units } & \multicolumn{3}{|c|}{ Levels } \\
\cline { 4 - 6 } & & & $-\mathbf{1}$ & $\boldsymbol{0}$ & $\boldsymbol{+ 1}$ \\
\hline $\mathbf{1}$ & $\boldsymbol{p H}$ & $\mathbf{p H}$ & 5.51 & 5.58 & 5.65 \\
\hline $\mathbf{2}$ & Temperature & ${ }^{\circ} \mathbf{C}$ & 44.4 & 48.59 & 52.78 \\
\hline $\mathbf{3}$ & Total pressure & $\mathbf{b a r}$ & 13.4 & 23.8 & 34.2 \\
\hline $\mathbf{4}$ & Shear stress & $\mathbf{P a}$ & 1 & 15 & 30 \\
\hline
\end{tabular}

\subsection{Method}

For the four variables the design required with 16 star points (cube points), eight axial points to form central composite design with seven center points for replication to estimate the experimental error. The design was generated and analyzed using MINITAB16 statistical package. The levels of each factor were chosen as $-1,0,+1$ in coded form to have a central composite design as shown in Table 3.

Table 3: Matrix of order and design of the experiments and test outputs

\begin{tabular}{|c|c|c|c|c|c|c|c|c|c|}
\hline 齐 & 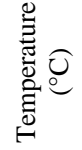 & 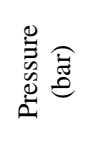 & I & 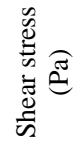 & 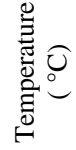 & 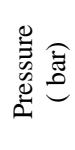 & $\frac{T}{2}$ & 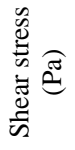 & 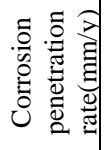 \\
\hline
\end{tabular}

www.ijsea.com 


\begin{tabular}{|l|c|c|c|c|c|c|c|c|c|}
\hline 1. & 0 & 0 & 0 & 0.05 & 48.59 & 23.8 & 5.58 & 16.2 & 2.30 \\
\hline 2. & 0 & 0 & 0 & 0 & 48.59 & 23.8 & 5.58 & 15.5 & 2.30 \\
\hline 3. & -1 & 1 & -1 & -1 & 44.40 & 34.2 & 5.51 & 1.00 & 3.07 \\
\hline 4. & 1 & -1 & 1 & -1 & 52.78 & 13.4 & 5.65 & 1.00 & 1.63 \\
\hline 5. & 0 & 0 & 0.05 & 0 & 48.59 & 23.8 & 5.58 & 15.5 & 2.29 \\
\hline 6. & 0 & 0 & -0.05 & 0 & 48.59 & 23.8 & 5.57 & 15.5 & 2.31 \\
\hline 7. & -1 & -1 & 1 & 1 & 44.40 & 13.4 & 5.65 & 30.0 & 2.08 \\
\hline 8. & -1 & 1 & -1 & 1 & 44.40 & 34.2 & 5.51 & 30.0 & 3.12 \\
\hline 9. & 0 & 0.05 & 0 & 0 & 48.59 & 24.32 & 5.58 & 15.5 & 2.33 \\
\hline 10. & -0.05 & 0 & 0 & 0 & 48.38 & 23.8 & 5.58 & 15.5 & 2.30 \\
\hline 11. & 0.05 & 0 & 0 & 0 & 48.79 & 23.8 & 5.58 & 15.5 & 2.30 \\
\hline 12. & 1 & -1 & -1 & -1 & 52.78 & 13.4 & 5.51 & 1.00 & 1.86 \\
\hline 13. & 0 & 0 & 0 & -0.05 & 48.59 & 23.8 & 5.58 & 14.8 & 2.30 \\
\hline 14. & 1 & 1 & 1 & 1 & 52.78 & 34.2 & 5.65 & 30.0 & 2.20 \\
\hline 15. & -1 & 1 & 1 & -1 & 44.40 & 34.2 & 5.65 & 1.00 & 2.72 \\
\hline 16. & -1 & -1 & -1 & 1 & 44.40 & 13.4 & 5.51 & 30.0 & 2.30 \\
\hline 17. & 0 & 0 & 0 & 0 & 48.59 & 23.8 & 5.58 & 15.5 & 2.30 \\
\hline 18. & 1 & -1 & -1 & 1 & 52.78 & 13.4 & 5.51 & 30.0 & 1.81 \\
\hline 19. & -1 & -1 & 1 & -1 & 44.40 & 13.4 & 5.65 & 1.00 & 1.38 \\
\hline 20. & 1 & -1 & 1 & 1 & 52.78 & 13.4 & 5.65 & 30.0 & 1.58 \\
\hline 21. & 0 & 0 & 0 & 0 & 48.59 & 23.8 & 5.58 & 15.5 & 2.30 \\
\hline 22. & 1 & 1 & -1 & 1 & 52.78 & 34.2 & 5.51 & 30.0 & 2.55 \\
\hline 23. & 0 & 0 & 0 & 0 & 48.59 & 23.8 & 5.58 & 15.5 & 2.30 \\
\hline 24. & 0 & -0.05 & 0 & 0 & 48.59 & 23.28 & 5.58 & 15.5 & 2.27 \\
\hline 25. & 1 & 1 & 1 & -1 & 52.78 & 34.2 & 5.65 & 1.00 & 2.90 \\
\hline
\end{tabular}

\begin{tabular}{|l|c|c|c|c|c|c|c|c|c|}
\hline 26. & -1 & 1 & 1 & 1 & 44.40 & 34.2 & 5.65 & 30.0 & 2.77 \\
\hline 27. & 0 & 0 & 0 & 0 & 48.59 & 23.8 & 5.58 & 15.5 & 2.30 \\
\hline 28. & 1 & 1 & -1 & -1 & 52.78 & 34.2 & 5.51 & 1.00 & 3.25 \\
\hline 29. & 0 & 0 & 0 & 0 & 48.59 & 23.8 & 5.58 & 15.5 & 2.30 \\
\hline 30. & 0 & 0 & 0 & 0 & 48.59 & 23.8 & 5.58 & 15.5 & 2.30 \\
\hline 31. & -1 & -1 & -1 & -1 & 44.40 & 13.4 & 5.51 & 1.00 & 1.60 \\
\hline
\end{tabular}

\section{DEVELOPMENT OF THE MODEL}

Fuzzy Logic (FL) is based on Fuzzy Set Theory that was established in 1965. In general, for any industrial control application, the information required for the initial analysis and design of the system is divided in two kinds: numerical information and linguistic information obtained from skilled human beings. These systems are then calibrated until results start producing answers closer to the original goal. Simulations are run to validate the system and prove its correct performance for the specific problem[12]. The types of information are different in nature but they both have a common characteristic; each is usually incomplete by itself. Although the system can be controlled successfully by a human operator, some of the information will be lost when transferring his or her experience onto an expert system structure. On the other hand, past numerical data is not enough to predict future control situations[7]. The investigation based on fuzzy-logic finds applications in unclear and undecided environment. In the recent research trends, fuzzy-logic-based multi-criteria decision making techniques have become very popular in doing optimization of different manufacturing processes. a Mamdani FIS thus providing the system's designer with more flexibility in the design of the system. However, it should be noted that the Mamdani FIS can be used directly for either MISO systems (multiple input single output) as well as for MIMO systems (multiple input multiple output), while the Sugeno FIS can only be used in MISO systems [8-9-10-11]. Fuzzy logic and the concept of linguistic variables have found a number of applications in such diverse fields as industrial process control, medical diagnosis, assessment of credit worthiness, risk analysis, etc. In present study of Mamdani a fuzzy control system is based on Fuzzy Logic, Mamdani type can be summarized all steps are shown in Figure 1. 


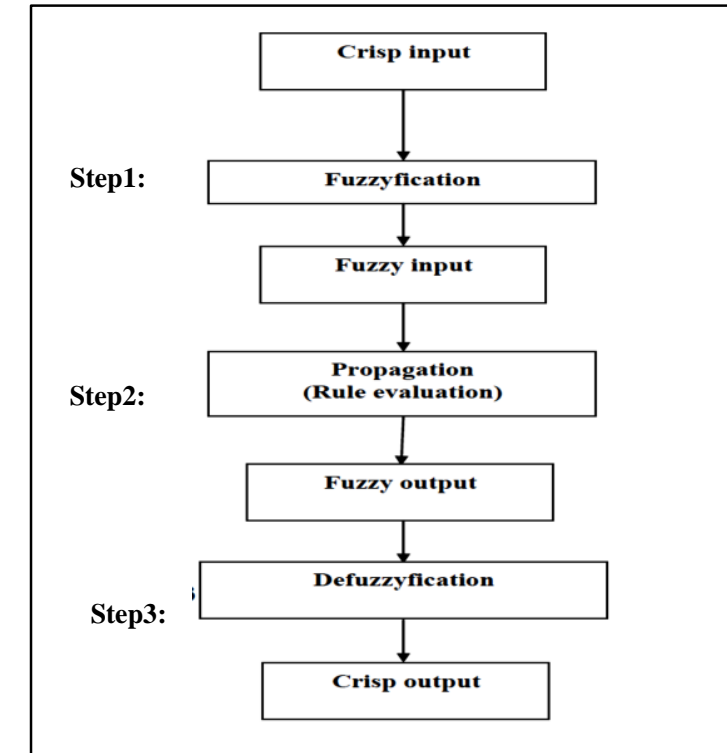

Figure 1: Steps of fuzzy control system

The structure of the four inputs and one outputs fuzzy logic controller developed for this study is shown in Figure 2.

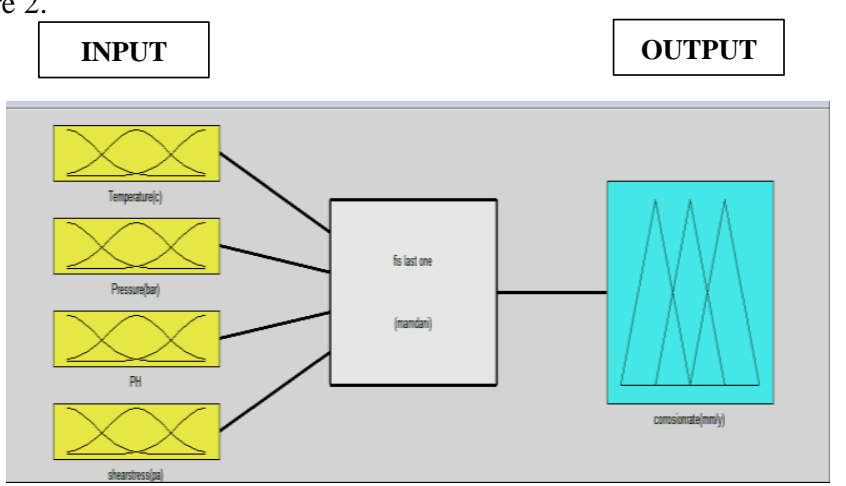

Figure 2: The four inputs-one output in "FIS Editor"

Four inputs and one output (CPR) fuzzy-logic system is used. The inference engine (Mamdani fuzzy inference system) performs fuzzy reasoning with fuzzy rules for generating a fuzzy value. These fuzzy rules are shown in the form of 'if-then' control rule. Temperature , pH, pressure, shear stress are inputs to the fuzzy logic system. The linguistic membership function for instance
Low, Medium, High and are used to represent of input variables. Likewise the output is being represented by the membership functions such as Very Low, Low, Medium, High, very High. The triangular and Gaussian shaped membership function, which is used in this study. The fuzzification of the four inputs e.g. Temperature, $\mathrm{pH}$, pressure and shear stress. The triangular membership function graph is shown to define how the values of the input and output (Y=CPR) are mapped to a value between 0 and 1 . The linguistic membership function such as Low, Medium And High are used to represent of input variables. Similarly, the output is represented by the membership functions such as Very Low, Low, Medium, High, very High, the Gaussian shaped membership function used in this work. Fuzzy logic 'if-then' control rule represented with 31 rules as shown in Table 4.

\section{Table 4: Fuzzy rule for corrosion penetration rate of API X52 composites}

Rule1. If (Temperature $\left({ }^{\circ} \mathrm{C}\right)$ is Med) and (Pressure(bar) is Med) and (pH is Med) and (Shear $\operatorname{Stress}(\mathrm{Pa})$ is Med) then (corrosion penetration rate $(\mathrm{mm} / \mathrm{y})$ is Low) $(0.45)$

Rule 2. If (Temperature $\left({ }^{\circ} \mathrm{C}\right)$ is Med) and (Pressure(bar) is Med) and (pH is Med) and (Shear $\operatorname{Stress}(\mathrm{Pa})$ is Med) then (corrosion penetration rate $(\mathrm{mm} / \mathrm{y})$ is Low) $(0.45)$

Rule 3. If (Temperature $\left({ }^{\circ} \mathrm{C}\right)$ is Low) and (Pressure(bar) is High) and ( $\mathrm{pH}$ is Low) and (Shear $\mathrm{Stress}(\mathrm{Pa})$ is Low) then (corrosion penetration rate $(\mathrm{mm} / \mathrm{y})$ is V Low) $(0.01)$

Rule 29. If (Temperature $\left({ }^{\circ} \mathrm{C}\right)$ is Med) and (Pressure(bar) is Med) and (pH is Med) and (Shear Stress $(\mathrm{Pa})$ is Med) then (corrosion penetration rate $(\mathrm{mm} / \mathrm{y})$ is Low) $(0.45)$

Rule 30. If (Temperature $\left({ }^{\circ} \mathrm{C}\right)$ is Med) and (Pressure(bar) is Med) and (pH is Med) and (Shear $\operatorname{Stress}(\mathrm{Pa})$ is Med) then (corrosion penetration rate $(\mathrm{mm} / \mathrm{y})$ is Low) $(0.45)$

Rule 31. If (Temperature $\left({ }^{\circ} \mathrm{C}\right)$ is Low) and (Pressure(bar) is Low) and ( $\mathrm{pH}$ is Low)and (Shear Stress $(\mathrm{Pa})$ is Low) then (corrosion penetration rate $(\mathrm{mm} / \mathrm{y})$ is V Low) $(0.15)$.

The fuzzy inputs are linguistically divided into three levels such as low, medium and high which is shown in Figure 3.

Figure 4 shows the fuzzy output linguistically divided into six levels such as low, medium, high and very high 


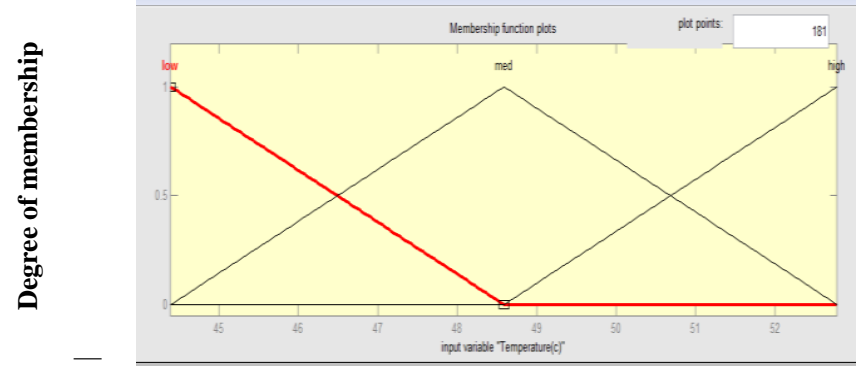

- (a)Temperature $\left({ }^{\circ} \mathrm{C}\right)$.

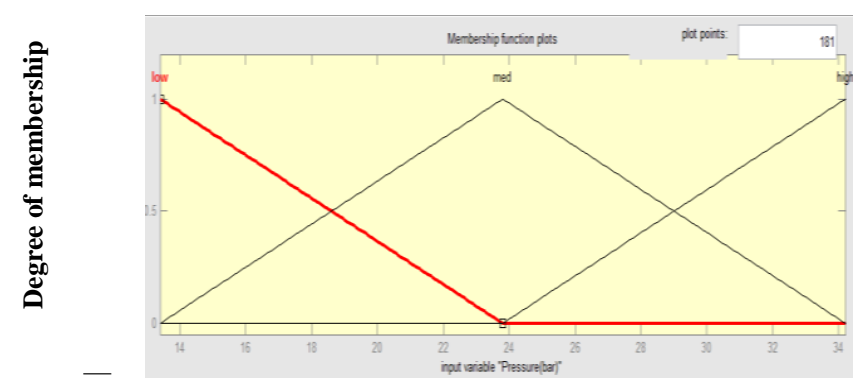

- (b) pressure(bar)

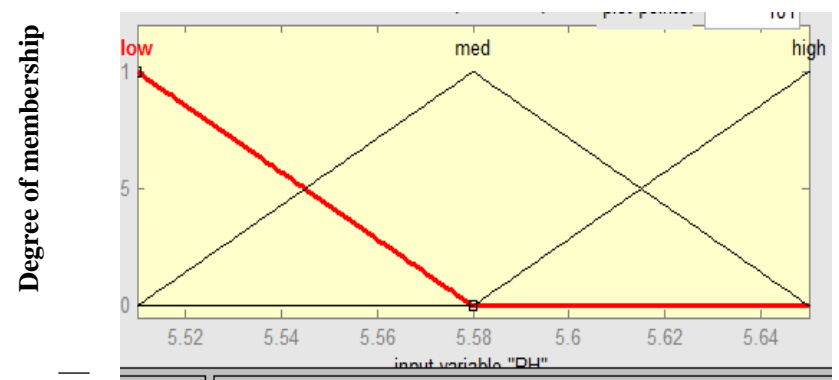

(c) $\mathrm{pH}$

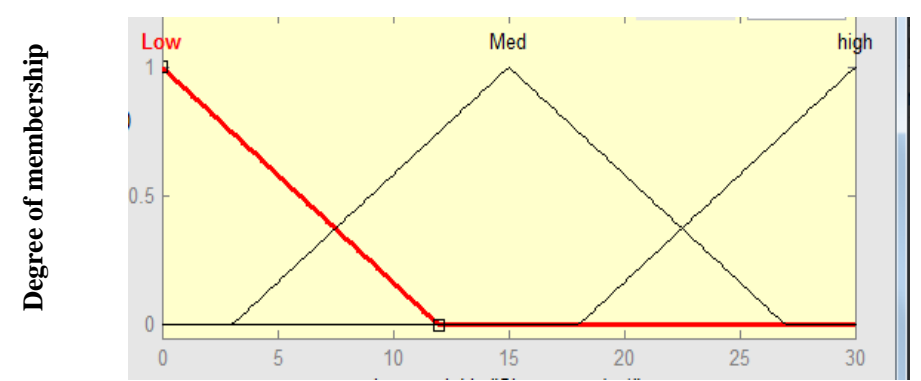

(d) Shear Stress $(\mathrm{Pa})$

Figure 3: Membership functions for inputs process parameters

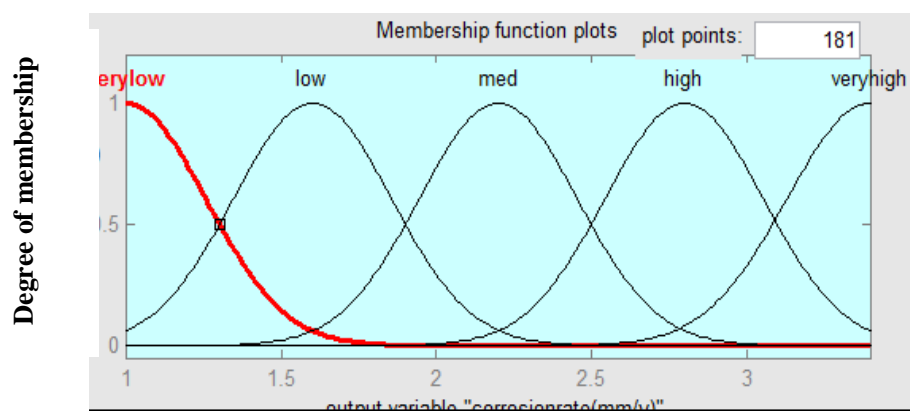

Figure 4: Membership functions for outputs: corrosion penetration rate(mm/y)

\section{SIMULATION OF LOGIC MODEL FUZZY}

In this study, the fuzzy model has been developed based on 31 experiments of corrosion penetration rate of process parameters. The fuzzy model was simulated for test cases which has been done within the range of the fuzzy set. The experiments was conducted for the three levels of each parameter. The purpose of the simulation was to minimize the error of outputs for test case experiments. A MATLAB Simulink model was developed to predict CPR. Moreover, to confirm the adequacy of fuzzy logic model, the predicted values of the corrosion rate predicted by using the proposed fuzzy model were compared with the predicted values of the corrosion rate by using RSM. These comparison are shown in Table 4.8 with their mean absolute error MAE. The MAE for 
the predicted of corrosion penetration rate by using the proposed fuzzy logic model is lower than the predicted of corrosion penetration rate by using RSM, the difference was observed to be $0.1269 \mathrm{~mm} / \mathrm{y}$. The results from fuzzy logic simulation indicated that the predicted values and experimental values closely agreed. In some cases, the predicted values and experimental values are observed to be little deviated. That might be due to some experimental error.

Table 5 : The experimental, predicted and mean absolute error of the corrosion penetration rate

\begin{tabular}{|c|c|c|c|c|c|}
\hline Exp. No & $\begin{array}{c}\text { Predicted CPR, } \\
\text { Using RSM } \\
,(\mathrm{mm} / \mathrm{y})\end{array}$ & $\begin{array}{l}\text { FL predicted } \\
\text { result }\end{array}$ & $\begin{array}{c}\text { Experimental CPR, } \\
\text { Using (Norsok) }\end{array}$ & $\begin{array}{l}\text { Error Using } \\
\quad \text { RSM }\end{array}$ & $\begin{array}{l}\text { Error Using } \\
\quad \text { Fuzzy }\end{array}$ \\
\hline 1 & 2.32 & 2.3 & 2.3 & 0.02 & 0 \\
\hline 2 & 2.30 & 2.3 & 2.3 & 0 & 0 \\
\hline 3 & 2.30 & 2.1 & 2.1 & 0.2 & 0 \\
\hline 4 & 1.52 & 1.2 & 1.0 & 0.52 & 0.2 \\
\hline 5 & 2.29 & 2.3 & 2.3 & 0.01 & 0 \\
\hline 6 & 2.31 & 2.3 & 2.3 & 0.01 & 0 \\
\hline 7 & 2.19 & 1.61 & 1.7 & 0.49 & 0.09 \\
\hline 8 & 3.88 & 3.2 & 3.4 & 0.48 & 0.2 \\
\hline 9 & 2.33 & 2.3 & 2.3 & 0.03 & 0 \\
\hline 10 & 2.30 & 2.3 & 2.3 & 0 & 0 \\
\hline 11 & 2.30 & 2.3 & 2.3 & 0 & 0 \\
\hline 12 & 1.68 & 1.2 & 1.2 & 0.48 & 0 \\
\hline 13 & 2.28 & 2.3 & 2.3 & 0.02 & 0 \\
\hline 14 & 2.90 & 2.71 & 2.7 & 0.2 & 0.01 \\
\hline 15 & 2.02 & 1.9 & 1.9 & 0.12 & 0 \\
\hline 16 & 2.48 & 1.9 & 2.0 & 0.48 & 0.1 \\
\hline 17 & 2.30 & 2.3 & 2.3 & 0 & 0 \\
\hline 18 & 1.98 & 1.8 & 1.8 & 0.18 & 0 \\
\hline 19 & 1.26 & 1.2 & 1.1 & 0.16 & 0.1 \\
\hline 20 & 1.70 & 1.61 & 1.6 & 0.1 & 0.01 \\
\hline 21 & 2.30 & 2.3 & 2.3 & 0 & 0 \\
\hline 22 & 3.31 & 3.19 & 3.2 & 0.11 & 0.01 \\
\hline 23 & 2.30 & 2.3 & 2.3 & 0 & 0 \\
\hline 24 & 2.27 & 2.3 & 2.3 & 0.03 & 0 \\
\hline 25 & 2.19 & 1.69 & 1.7 & 0.49 & 0.01 \\
\hline 26 & 3.47 & 3 & 3.0 & 0.47 & 0 \\
\hline 27 & 2.30 & 2.3 & 2.3 & 0 & 0 \\
\hline 28 & 2.48 & 2.2 & 2.0 & 0.48 & 0.2 \\
\hline 29 & 2.30 & 2.3 & 2.3 & 0.02 & 0 \\
\hline 30 & 2.30 & 2.3 & 2.3 & 0 & 0 \\
\hline 31 & 1.43 & 1.3 & 1.3 & 0.2 & 0 \\
\hline & & & MAE & 0.1814 & 0.0332 \\
\hline
\end{tabular}

\section{CONCLUSION}

During this study, attempts were done to predict the corrosion penetration rates of the pipelines that are used for transporting the crude oil between Tobruk and Sarir stations. The corrosion values were measured by using Norsok-506 standard software. The theory and principles of corrosion were also explained. Conclusions can be summarized as follow:

1. Based on ANOVA analysis, the temperature, pressure, $\mathrm{pH}$ and shear stress had significant effect on corrosion rate, while the quadratic effect was insignificant. However, the interaction between (temperature and shear stress), (pressure and $\mathrm{pH}$ ), (pressure and shear stress) and ( $\mathrm{pH}$ and shear stress) had the more significant effect on corrosion penetration rate

2. Based on comparison between models that were developed by using fuzzy logic and RSM technique, it was concluded that, models based on fuzzy logic were better with mean absolute error of $0.0412 \mathrm{~mm} / \mathrm{y}$ with in comparison $0.1681 \mathrm{~mm} / \mathrm{y}$ for RSM.

3. The optimal values for the numerically calculated CPR using the fuzzy logic model with the formula using Root- Sum Square, it was found that $\mathrm{CPR}=2.16 \mathrm{~mm} / \mathrm{y}$, temperature $=44.4^{\circ} \mathrm{C}$, pressure $=34.28 \mathrm{~Pa}, \mathrm{pH}=5.51$ and shear stress $=1 \mathrm{bar}$.

4. The study proved that there was other technique can give results close to real data instead of Norsok software which spends million dollars.

\section{ACKNOWLEDGMENTS}

First of all, I would like to express my sincere thanks and gratitude to Almighty Allah for such an opportunity in my life and thanks are due to the engineers in corrosion department at Arabian Gulf Oil Company, Benghazi (Libya) and thanks to everyone help me for support to complete the study.

\section{REFERENCES}

[1] . Song, F.M., Kirk, D.W., Graydon, J.W., and Cormack D.E (2004) " Predicting carbon dioxide corrosion of bare steel under an aqueous boundary layer". Corrosion, 60 , pp. 736- 743 . 
[2]. Mikhailovskii, Y.N., Marshakov, A.I., and Petrov, N.A (1997) Monitoring of underground pipeline corrosion condition with sensory instruments. Prot. Met., 33, pp. 293-295.

[3]. Lopez, D.A., Schreiner, W.H., de Sanchez, S.R., and Simison, S.N. (2003) " The influence of carbon steel microstructure on corrosion layers: an XPS and SEM characterization". Appl. Surf. Sci., 207, pp. 69-85.

[4]. Fu, S.L., Garcia, J.G., and Griffin, A.M. (1996) " Corrosion Resistance of Some Downhole Tubing Materials and Inhibitor Effectiveness in Sweet Environments". Proceedings of CORROSION / 1996, NACE International, Houston, Taxes, Paper no. 21.

[5]. Kermani, M.B., and Morshed, A. (2003) "Carbon dioxide corrosion in oil and gas roduction - A compendium". Corrosion, 59, pp. 659-683.

[6]. Villamizar, W., Casales, M., Gonzalez-Rodriguez, J.G., and Martinez, L. (2007) " $\mathrm{CO}_{2}$ corrosion inhibition by hydroxyethyl, aminoethyl, and amidoethyl imidazolines in water-oil mixtures". J. Solid State Electrochem., 11, pp. 619-629.

[7] luis alfonso," LEANRNING FUZZY LOGIC FROM EXAMPLES" ". [Thesis]. Ohio University; March 1994.

[8] E. H. Mamdani and S. Assilian, "An experiment in linguistic synthesis with a fuzzy logic controller", International Journal of Man-Machine Studies, Vol. 7, No.1,pp. 1-13, 1975 .

[9] T. Takagi and M. Sugeno, "Fuzzy identification of systems and its applications to modeling and control", IEEE Trans, on Systems, Man and Cybernetics, 15, pp. 116$132,1985$.

[10] Jassbi, J.J.; Serra, P.J.A.; Ribeiro, R.A.; Donati, A.; "A Comparison of Mamdani and Sugeno Inference Systems for a space Fault Detection Application" Automation Congress, 2006. WAC '06. World. Issue Date: 24-26 July ,On page(s): 1 - 8,2006.

[11] J. Mendel, "Uncertain rule-based fuzzy inference systems: Introduction and new directions", Prentice-Hall, 2001.

[12] Mamdani, E.H. and S. Assilian, "An experiment in linguistic synthesis with a fuzzy logic controller," International Journal of Man-Machine Studies, Vol. 7, No. 1, pp. 1$13,1975$. 